\title{
ON THE SPECTRA OF PISOT NUMBERS
}

\author{
TOUFIK ZAIMI \\ Département de mathématiques Université Larbi Ben M'hidi Oum El Bouaghi 04000, Algérie \\ e-mail:toufikzaimi@yahoo.com
}

(Received 16 March 2010; revised 14 August 2010; accepted 11 June 2011)

\begin{abstract}
Let $\theta$ be a real number greater than 1, and let (( )) be the fractional part function. Then, $\theta$ is said to be a $Z$-number if there is a non-zero real number $\lambda$ such that $\left(\left(\lambda \theta^{n}\right)\right)<\frac{1}{2}$ for all $n \in \mathbb{N}$. Dubickas (A. Dubickas, Even and odd integral parts of powers of a real number, Glasg. Math. J., 48 (2006), 331-336) showed that strong Pisot numbers are $Z$-numbers. Here it is proved that $\theta$ is a strong Pisot number if and only if there exists $\lambda \neq 0$ such that $((\lambda \alpha))<\frac{1}{2}$ for all $\alpha \in\left\{\theta^{n} \mid n \in \mathbb{N}\right\} \cup\left\{\sum_{n=0}^{N} \theta^{n} \mid N \in \mathbb{N}\right\}$. Also, the following characterisation of Pisot numbers among real numbers greater than 1 is shown: $\theta$ is a Pisot number $\Leftrightarrow \exists \lambda \neq 0$ such that $\|\lambda \alpha\|<\frac{1}{3}$ for all $\alpha \in\left\{\sum_{n=0}^{N} a_{n} \theta^{n} \mid\right.$ $\left.a_{n} \in\{0,1\}, N \in \mathbb{N}\right\}$, where $\|\lambda \alpha\|=\min \{((\lambda \alpha)), 1-((\lambda \alpha))\}$.

2000 Mathematics Subject Classification. 11R80, 11J71, 11R06.
\end{abstract}

1. Introduction. For a point $t$ of the real line $\mathbb{R}$ we denote by $[t]$ the largest element of the ring $\mathbb{Z}$ of rational integers, not exceeding $t$. We also denote by $((t))$ and $\|t\|$ the difference $t-[t]$ and the minimum of the set $\{((\lambda \alpha)), 1-((\lambda \alpha))\}$, respectively. Namely, $[t]$ is the integer part of $t,(())$ is the fractional part function and $\|t\|$ is the usual distance from $t$ to $\mathbb{Z}$.

Let throughout $\theta \in(1, \infty), \lambda \in \mathbb{R} \backslash\{0\}$ and $n \in \mathbb{N}:=\mathbb{Z} \cap[0, \infty)$. Dubickas in [3] defined a subset $Z$ of $(1, \infty)$, with the property that for each $\theta \in Z$, there is $\lambda=\lambda(\theta)$ such that $\left(\left(\lambda \theta^{n}\right)\right)<\frac{1}{2}$ for all $n$. An element of $Z$ is called a $Z$-number. A result due to Tijdeman, and cited in [3] gives immediately that $[3, \infty) \subset Z$. Set $Y:=(1, \infty) \backslash Z$. Some classes of algebraic integers, which belong to $Z \cap(1,3)$, or to $Y \cap(1,2)$, are exhibited in [3], and from this one can easily deduce that 2 is a left-hand limit point of $Z$, and 1 is a limit point of $Y$. Dubickas proved in particular that strong Pisot numbers are $Z$-numbers. Recall that a Pisot number is a real algebraic integer greater than 1 whose other conjugates are of modulus less than 1 . The set of Pisot numbers is usually noted $S$. A Pisot number $\theta$ of degree $d$ is called a strong Pisot number if $d=1$, or if $d \geq 2$ and $\theta$ has a conjugate belonging to the interval $(0,1)$, which is greater than the absolute values of $d-2$ remaining conjugates of $\theta$ [2]. We denote by $S_{s t}$ the set of strong Pisot numbers.

Let

$$
\begin{gathered}
A_{0}=A_{0}(\theta):=\left\{\theta^{n} \mid n \in \mathbb{N}\right\} \cup\left\{\sum_{n=0}^{N} \theta^{n} \mid N \in \mathbb{N}\right\}, \\
A_{m}=A_{m}(\theta):=\left\{\sum_{n=0}^{N} a_{n} \theta^{n} \mid a_{n} \in\{0, \ldots, m\}, N \in \mathbb{N}\right\},
\end{gathered}
$$


where $m \in \mathbb{N} \backslash\{0\}$, and

$$
B_{m}=B_{m}(\theta):=A_{m}(\theta)-A_{m}(\theta) .
$$

The first aim of this note is to show that strong Pisot numbers are a kind of 'strong $Z$-numbers' :

THEOREM 1. The following are equivalent.

(i) $\theta \in S_{s t}$.

(ii) For any $\varepsilon>0$ and any $m$, there is $\lambda$ such that $((\lambda \alpha))<\varepsilon$ for all $\alpha \in A_{m}(\theta)$.

(iii) There exist $m$ and $\lambda$ such that $((\lambda \alpha))<\frac{1}{2}$ for all $\alpha \in A_{m}(\theta)$.

(iv) There is $\lambda$ such that $((\lambda \alpha))<\frac{1}{2}$ for all $\alpha \in A_{0}(\theta)$.

In terms of fractional part function, Theorem 1 may be viewed as a characterisation of strong Pisot numbers among real numbers greater than 1 . This contrasts with the famous characterisation of Pisot numbers among real numbers, due to Pisot [4], which says: If there is $\lambda$ such that $\sum_{n \in \mathbb{N}}\left\|\lambda \theta^{n}\right\|^{2}<\infty$, then $\theta \in S$. The important question whether there is a transcendental number $\theta$ satisfying $\lim _{n \rightarrow \infty}\left\|\lambda \theta^{n}\right\|=0$ for some $\lambda$, is still unsolved [1]. We shall mainly use this last mentioned result of Pisot to prove Theorem 1 and the result below:

THEOREM 2. The following are equivalent.

(i) $\theta \in S$.

(ii) For any $\varepsilon>0$ and any $m \in \mathbb{N}$, there is $\lambda$ such that $\|\lambda \beta\|<\varepsilon$ for all $\beta \in B_{m}(\theta)$.

(iii) For any $\varepsilon>0$ and any $m \in \mathbb{N}$, there is $\lambda$ such that $\|\lambda \alpha\|<\varepsilon$ for all $\alpha \in A_{m}(\theta)$.

(iv) There is $\lambda$ such that $\|\lambda \alpha\|<\frac{1}{3}$ for all $\alpha \in A_{1}(\theta)$.

In these pages when we speak about conjugates, minimal polynomial and degree of an algebraic number we mean over the field of the rationals $\mathbb{Q}$. For a Pisot number $\theta$ of degree $d$, we denote by $\theta:=\theta_{1}, \ldots, \theta_{d}$, the conjugates of $\theta$, and by $\sigma_{1}, \ldots, \sigma_{d}$, the embeddings of $\mathbb{Q}(\theta)$ into the complex field $\mathbb{C}$, where $\sigma_{1}$ is the identity of $\mathbb{Q}(\theta)$. As usual, for an element $\alpha$ of the field $\mathbb{Q}(\theta)$, we denote by $\operatorname{Trace}(\alpha)$ the sum $\sigma_{1}(\alpha)+\cdots+\sigma_{d}(\alpha)$, namely the trace of $\alpha$ for the extension $\mathbb{Q}(\theta) / \mathbb{Q}$. The proofs of Theorems 1 and 2 appear in the following sections, consecutively. It is interesting to determine whether the constant $1 / 3$ in Theorem 2(iv) is optimal, or whether we may replace $A_{1}(\theta)$ by one of its proper subsets without affecting the conclusion. Analogue questions may be posed for Theorem 1. Distribution in $\mathbb{R}$ of the elements of the set $S_{s t}$ is another problem related to Theorem 1. Some computations suggest the following conjecture: $\min S_{s t}=2, \min \left(S_{s t} \backslash\{2\}\right)=(3+\sqrt{5}) / 2$ and $\min S_{s t}^{\prime}=3$, where $S_{s t}^{\prime}$ is the derived set of $S_{s t}$. From the proof of the result below, one can easily deduce that 3 is a left-hand limit point of $S_{s t}$.

Proposition. The set $S_{s t}^{\prime}$ contains $\mathbb{N} \cap[3, \infty)$.

Proof. Let $b$ be a rational integer greater than 2 and let $P_{n}(x):=x^{n}(x-b)+1$, where $n \geq b$. Since $\left|b z^{n}\right|=b>2 \geq\left|z^{n+1}+1\right|$ when the complex number $z$ runs through the unit circle, Rouche's theorem gives that $P_{n}$ has $n$ roots with modulus less than 1 , and so the polynomial $P_{n}$ has a unique root, say $\theta_{(n)}$, of modulus greater than 1 , as $P_{n}(0)=1$. Hence, $P_{n}$ is irreducible over $\mathbb{Q}$ and is the minimal polynomial of $\theta_{(n)}$. Notice also that the real function $P_{n}(t)$ is decreasing on the interval $(0, n b /(n+1))$ and is increasing on $(n b /(n+1), \infty)$ because its formal derivative is $(n+1) t^{n-1}(t-n b /(n+1))$. It follows by the relations $P_{n}(0)=1, P_{n}(1)=2-b, b-1<n b /(n+1)$ and $P_{n}(b)=1$ that $P_{n}$ 
has a unique root, say $\rho$, in the interval $(0,1)$ and $\theta_{(n)} \in(b-1, b)$. Consequently, $\theta_{(n)}$ is a Pisot number, and

$$
\lim _{n \rightarrow \infty} \theta_{(n)}=b,
$$

since $0<b-\theta_{(n)}=\frac{1}{\theta_{(n)}^{n}}<\frac{1}{(b-1)^{n}} \leq \frac{1}{2^{n}}$. If $\alpha$ is a conjugate of $\theta_{(n)}$ such that $\alpha \neq \theta_{(n)}$, then $b|\alpha|^{n}=\left|\alpha^{n+1}+1\right| \leq|\alpha|^{n+1}+1$; hence $P(|\alpha|)=|\alpha|^{n+1}-b|\alpha|^{n}+1 \geq 0$ and so $|\alpha| \leq \rho$, as $P_{n}(\rho)=0$ and $P_{n}(t)$ is decreasing on $(0,1)$. Moreover, the equality $|\alpha|=\rho$ holds only if $\left|\alpha^{n+1}+1\right|=|\alpha|^{n+1}+1$ that is when $\alpha^{n+1}$ is a positive real number. It follows in this case by the equality $\alpha^{n+1}=b \alpha^{n}-1$ that $\alpha^{n}>1 / b>0$, and so $\alpha=\alpha^{n+1} / \alpha^{n}$ is also a positive real number; thus, $\alpha=\rho$ and so $\theta_{(n)} \in S_{s t}$.

REMARK. A simple computation shows that any polynomial of the form $x^{2}-$ $b x+k$, where $b \in \mathbb{N} \cap[3, \infty[$ and $k \in\{1, \ldots, b-2\}$, is the minimal polynomial of a quadratic strong Pisot number, say $\theta_{k}$, satisfying $b-1<\theta_{k}<b$. Similarly, as in the above proof, by considering the sequence of polynomials $x^{n}\left(x^{2}-b x+k\right)+1$, we easily obtain that $\theta_{k}$ is a left-hand limit point of the set $S_{s t}$, when $b \geq 4$ and $k \leq b-3$. Consequently, each interval of the form $[n, n+1]$, where $n \geq 3$, contains at least $n$ elements of the set $S_{s t}^{\prime}$.

2. Proof of Theorem 1. To make clear the proof of Theorem 1, let us recall some results on Pisot numbers. The first two results are due to Pisot [4] and Smyth [6].

LEMMA 1. ([4]) If $\sum_{n=0}^{\infty}\left\|\lambda \theta^{n}\right\|^{2}<\infty$ for some $\lambda$, then $\theta \in S$ and $\lambda \in \mathbb{Q}(\theta)$.

Lemma 2. ([6]) Two distinct conjugates of a Pisot number having the same modulus are complex conjugates.

Theorem (ii) and Lemma 2 of [7] yield the following :

LEMMA 3. If $\lambda$ satisfies $\lim _{n \rightarrow \infty}\left\|\lambda \theta^{n}\right\|=0$ for some $\theta \in S$, then $\lambda \in \mathbb{Q}(\theta)$ and there is $N \in \mathbb{N}$ such that Trace $\left(\lambda \theta^{n}\right) \in \mathbb{Z}$ for all $n \geq N$.

Finally, let us show a simple argument on the conjugates of a Pisot number.

LemMa 4. Let $\theta$ be a Pisot number of degree $d$. Then for any positive rational integer $p, \theta^{p}$ is a Pisot number of degree $d$. If $\rho e^{i a \pi}$ is a conjugate of $\theta$, where $i^{2}=-1$ and $(\rho, a) \in(0,1) \times(0,1)$, then for any $b \in \mathbb{R}$, the sequence $(((n a+b)))_{n}$ is dense in $[0,1]$.

Proof. Let $p$ be a positive rational integer. Then, $\theta^{p} \in \mathbb{Q}(\theta)$, and the conjugates of $\theta^{p}$ are among the numbers $\theta^{p}, \theta_{2}^{p}, \ldots, \theta_{d}^{p}$. Since $\left|\theta_{k}^{p}\right|<1$ for all $k \in\{2, \ldots, p\}, \theta^{p}$ is not repeated by the action of embeddings $\sigma_{1}, \ldots, \sigma_{d}$; thus $\mathbb{Q}(\theta)=\mathbb{Q}\left(\theta^{p}\right)$ and $\theta^{p}$ is a Pisot number of degree $d$. Let $\rho e^{i a \pi}$ be a non-real conjugate of $\theta$, then $\rho e^{-i a \pi}$ is also another conjugate of $\theta$, and so (by the first part of Lemma 4) $\rho^{p} e^{i \text { рал }}$ and $\rho^{p} e^{-i \text { рал }}$ are two distinct conjugates of $\theta^{p}$. Hence, $a \notin \mathbb{Q}$, and the result follows immediately by Kronecker's theorem (see for instance Appendix 8 in [5]).

Proof of Theorem 1. Let $\theta$ be a strong Pisot number with degree $d$, and let $\varepsilon>0$. If $d=1$, then $A_{m} \subset \mathbb{N}$ and so $((\alpha))=0<\varepsilon$ for all $\alpha \in A_{m}$. Now, suppose $d \geq 2$, and $\theta_{2}>\left|\theta_{3}\right| \geq \ldots \geq\left|\theta_{d}\right|$. Then, $t_{n}:=\operatorname{Trace}\left(\theta^{n}\right)=\theta^{n}+\theta_{2}^{n}+\cdots+\theta_{d}^{n} \in \mathbb{Z}, \theta_{2}^{n}+\cdots+$ $\theta_{d}^{n}=t_{n}-\theta^{n} \in \mathbb{R}, \theta_{2}^{n}+\cdots+\theta_{d}^{n}<d \theta_{2}^{n}$ and $\lim _{n \rightarrow \infty} \sum_{k=2}^{d}\left(\frac{\theta_{k}}{\theta_{2}}\right)^{n}=1$. Let $n_{1}$ be a positive 
rational integer such that for all $n \geq n_{1}$ we have

$$
0<\theta_{2}^{n}+\cdots+\theta_{d}^{n}
$$

and

$$
\frac{m d \theta_{2}^{n}}{1-\theta_{2}}<\min \{1, \varepsilon\} .
$$

Setting $\lambda:=-\theta^{n_{1}}$, we have $\lambda \theta^{n}=-\theta^{n+n_{1}}=-t_{n+n_{1}}+\theta_{2}^{n+n_{1}}+\cdots+\theta_{d}^{n+n_{1}}$, and the relations (1) and (2) give $0<\theta_{2}^{n+n_{1}}+\cdots+\theta_{d}^{n+n_{1}}<d \theta_{2}^{n+n_{1}^{2}}<\left(1-\theta_{2}\right) / m<1$ for all $n$. Hence, $-t_{n+n_{1}}=\left[\lambda \theta^{n}\right],\left(\left(\lambda \theta^{n}\right)\right)=\theta_{2}^{n+n_{1}}+\cdots+\theta_{d}^{n+n_{1}}$, and so $\left(\left(\lambda \theta^{n}\right)\right)<\left(1-\theta_{2}\right) \varepsilon / m<$ $\varepsilon$ for all $n$. Similarly, if $\alpha=\sum_{n=0}^{N} a_{n} \theta^{n}$, where $a_{n} \in\{0,1, \ldots, m\}$ and $N \in \mathbb{N}$, then $\lambda \alpha=$ $\sum_{n=0}^{N} a_{n} \lambda \theta^{n}=-\sum_{n=0}^{N} a_{n} t_{n+n_{1}}+\sum_{n=0}^{N} a_{n}\left(\theta_{2}^{n+n_{1}}+\cdots+\theta_{d}^{n+n_{1}}\right)$, and the inequalities (1) and (2) again yield $0<\sum_{n=0}^{N} a_{n}\left(\theta_{2}^{n+n_{1}}+\cdots+\theta_{d}^{n+n_{1}}\right)<\sum_{n=0}^{N} a_{n}\left(d \theta_{2}^{n+n_{1}}\right) \leq$ $m d \theta_{2}^{n_{1}} \sum_{n=0}^{N} \theta_{2}^{n}<\frac{m d \theta_{2}^{n_{1}}}{1-\theta_{2}}<\min \{1, \varepsilon\}$; thus, $((\lambda \alpha))=\sum_{n=0}^{N} a_{n}\left(\theta_{2}^{n+n_{1}}+\cdots+\theta_{d}^{n+n_{1}}\right)<\varepsilon$, as $-\sum_{n=0}^{N} a_{n} t_{n+n_{1}} \in \mathbb{Z}$, and so Theorem 1(ii) holds. The implications (ii) $\Longrightarrow$ (iii) $\Longrightarrow$ (iv) in Theorem 1 are trivially true, since $A_{0} \subset A_{1} \subset A_{m}$. To show that the proposition (iv) $\Longrightarrow$ (i), is true, let us first verify the equalities

$$
\sum_{n=0}^{N}\left(\left(\lambda \theta^{n}\right)\right)=\left(\left(\lambda \sum_{n=0}^{N} \theta^{n}\right)\right),
$$

where $\lambda$ satisfies $((\lambda \alpha))<\frac{1}{2}$ for all $\alpha \in A_{0}$, and $N \in \mathbb{N}$. It is clear that (3) holds for $N=$ 0 . By the relations $\lambda \sum_{n=0}^{N+1} \theta^{n}=\left[\lambda \sum_{n=0}^{N} \theta^{n}\right]+\left(\left(\lambda \sum_{n=0}^{N} \theta^{n}\right)\right)+\left[\lambda \theta^{N+1}\right]+\left(\left(\lambda \theta^{N+1}\right)\right)$ and $0 \leq\left(\left(\lambda \sum_{n=0}^{N} \theta^{n}\right)\right)+\left(\left(\lambda \theta^{N+1}\right)\right)<\frac{1}{2}+\frac{1}{2}$, where $N \in \mathbb{N}$, we have $\left(\left(\lambda \sum_{n=0}^{N+1} \theta^{n}\right)\right)=$ $\left(\left(\lambda \sum_{n=0}^{N} \theta^{n}\right)\right)+\left(\left(\lambda \theta^{N+1}\right)\right)$, and a simple induction gives (3). Letting $N$ tends to infinity in (3), we obtain

$$
\sum_{n=0}^{\infty}\left(\left(\lambda \theta^{n}\right)\right) \leq \frac{1}{2}
$$

and so $\sum_{n=0}^{\infty}\left\|\lambda \theta^{n}\right\| \leq \frac{1}{2}$. It follows by Lemma 1 that $\theta \in S$ and $\lambda \in \mathbb{Q}(\theta)$. The last inequality also gives $\lim _{n \rightarrow \infty}\left\|\lambda \theta^{n}\right\|=0$, and so by Lemma 3, there is $n_{2} \in \mathbb{N}$ such that $t_{n}:=\operatorname{Trace}\left(\lambda \theta^{n}\right) \in \mathbb{Z}$ for all $n \geq n_{2}$. Let $d$ be the degree of $\theta$. If $d=1$, then $\theta \in S_{s t}$. Suppose $d \geq 2$, and $\left|\theta_{2}\right| \geq \ldots \geq\left|\theta_{d}\right|$. Lemma 2 says that we have to prove that $\theta_{2}$ is a positive real number. Assume that $n \geq n_{2}$. Then, $t_{n}=\lambda \theta^{n}+\lambda_{2} \theta_{2}^{n}+\cdots+\lambda_{d} \theta_{d}^{n}$, where $\lambda_{k}=\sigma_{k}(\lambda)$ for $k \in\{1, \ldots, d\}, \lambda_{2} \theta_{2}^{n}+\cdots+\lambda_{d} \theta_{d}^{n}=t_{n}-\lambda \theta^{n} \in \mathbb{R}$, and

$$
t_{n}-\left[\lambda \theta^{n}\right]=\left(\left(\lambda \theta^{n}\right)\right)+\lambda_{2} \theta_{2}^{n}+\cdots+\lambda_{d} \theta_{d}^{n} .
$$

Let $n_{3}$ be the smallest element of $\mathbb{N}$ satisfying

$$
d\left|\theta_{2}\right|^{n_{3}} \max _{2 \leq j \leq d}\left|\lambda_{j}\right|<1 / 2
$$

Then, (4) gives, for $n \geq \max \left\{n_{2}, n_{3}\right\}$,

$$
-1 / 2<t_{n}-\left[\lambda \theta^{n}\right]<1 / 2+1 / 2,
$$


since $\left|\lambda_{2} \theta_{2}^{n}+\cdots+\lambda_{d} \theta_{d}^{n}\right|<d\left|\theta_{2}\right|^{n} \max _{1 \leq j \leq d}\left|\lambda_{j}\right|$ (recall that $0 \leq\left(\left(\lambda \theta^{n}\right)\right)<1 / 2$ for all $n)$; thus $t_{n}-\left[\lambda \theta^{n}\right]=0$ and so

$$
\lambda_{2} \theta_{2}^{n}+\cdots+\lambda_{d} \theta_{d}^{n}=-\left(\left(\lambda \theta^{n}\right)\right) \leq 0
$$

Now we claim that the result follows directly by (5) and Lemmas 2 and 4. Indeed, if $\theta_{2} \notin \mathbb{R}$, then $d \geq 3, \theta_{3}=\overline{\theta_{2}}$ and $\lambda_{3}=\overline{\lambda_{2}}$. Set $\theta_{2}:=\rho e^{i a \pi}$ and $\lambda_{2}=\eta e^{i b \pi}$, where $i^{2}=-1,(|a|, \rho) \in(0,1) \times(0,1), b \in(-1,1]$ and $\eta>0$. Then, Lemma 4 states that there are infinitely many $n$ such that the corresponding quantities $2 \eta \cos ((n a+b) \pi)+$ $\sum_{k=4}^{d} \lambda_{k} \frac{\theta_{k}^{n}}{\rho^{n}}$ are all positive because $\lim _{n \rightarrow \infty} \frac{\theta_{k}^{n}}{\rho^{n}}=0$ for all $k \in\{4, \ldots, d\}$; this leads to a contradiction since by (5) we have $\rho^{n}\left(2 \eta \cos ((n a+b) \pi)+\sum_{k=4}^{d} \lambda_{k} \frac{\theta_{k}^{n}}{\rho^{n}}\right)=\lambda_{2} \theta_{2}^{n}+$ $\lambda_{3} \theta_{3}^{n}+\cdots+\lambda_{d} \theta_{d}^{n} \leq 0$. Finally, if $\theta_{2} \in \mathbb{R}$, then the relation (5), together with Lemma 2, again gives $\lim _{n \rightarrow \infty} \frac{-\left(\left(\lambda \theta^{n}\right)\right)}{\theta_{2}^{n}}=\lambda_{2}$, and so $\theta_{2}>0$ and $\lambda_{2}<0$, as $\lambda=\sigma_{2}^{-1}\left(\lambda_{2}\right)=0$ when $\lambda_{2}=0$; thus $\theta \in S_{s t}$.

3. Proof of Theorem 2. Let $\varepsilon>0$, and let $\beta=\sum_{n=0}^{N} b_{n} \theta^{n}$, where $\theta$ is a Pisot number of degree $d, N \in \mathbb{N}$ and $b_{n} \in\{-m,-m+1, \ldots, m\}$. If $d=1$, then $B_{m} \subset \mathbb{N}$ and so $\|\beta\|=0<\varepsilon$. Suppose $d \geq 2$. It is clear that $\beta$ is an integer of the field $\mathbb{Q}(\theta)$, the conjugates of $\beta$ are among the numbers $\beta_{k}:=\sigma_{k}(\beta)=\sum_{n=0}^{N} b_{n} \theta_{k}^{n}$, where $k \in\{1, \ldots, d\}$, and

$$
\left|\beta_{k}\right| \leq m \sum_{n=0}^{N}\left|\theta_{k}\right|^{n}<\frac{m}{1-\left|\theta_{k}\right|} \text { for } k \in\{2, \ldots, d\} .
$$

Set $\lambda:=\theta^{p}$, where $p \in \mathbb{N}$ and satisfies $\left|\theta_{k}\right|^{p}<\frac{\varepsilon\left(1-\left|\theta_{k}\right|\right)}{m(d-1)}$ for all $k \in\{2, \ldots, d\}$. Then, $t:=\operatorname{Trace}(\lambda \beta)=\theta^{p} \beta+\theta_{2}^{p} \beta_{2}+\cdots+\theta_{d}^{p} \beta_{d} \in \mathbb{Z}$, and by the relation (6) we obtain

$$
\|\lambda \beta\| \leq|\lambda \beta-t|=\left|\theta_{2}^{p} \beta_{2} \cdots+\theta_{d}^{p} \beta_{d}\right|<\varepsilon ;
$$

thus, Theorem 2(ii) holds. The implications (ii) $\Rightarrow$ (iii) $\Rightarrow$ (iv) in Theorem 2 are trivially true because $A_{1} \subset A_{m} \subset B_{m}$. Now assume that there is $\lambda$ such that $\|\lambda \alpha\|<\frac{1}{3}$ for all $\alpha \in A_{1}$. We shall use Lemma 1 to prove that $\theta \in S$. Set $\lambda \theta^{n}:=x_{n}+y_{n}$, where $x_{n} \in \mathbb{Z}$ and $\left|y_{n}\right|=\left\|\lambda \theta^{n}\right\|$. If $s_{N}=\sum_{n=0}^{N} a_{n} \theta^{n}$, where $a_{n} \in\{0,1\}$ and $N \in \mathbb{N}$, then $\lambda s_{N}=$ $\sum_{n=0}^{N} a_{n} x_{n}+\sum_{n=0}^{N} a_{n} y_{n}$ and $\sum_{n=0}^{N} a_{n} x_{n} \in \mathbb{Z}$. Similarly, as in the proof of Theorem 1 , let us show the relation

$$
\left|\sum_{n=0}^{N} a_{n} y_{n}\right|<\frac{1}{3} \text { for all } N
$$

If $N=0$, then $a_{0} y_{0} \in\left\{0, y_{0}\right\}$, and so $-1 / 3<a_{0} y_{0}<1 / 3$, as $\left|y_{0}\right|=\|\lambda\|$. Suppose that (7) holds for some $N \in \mathbb{N}$, and let $s_{N+1}=\sum_{n=0}^{N+1} a_{n} \theta^{n}$, where $\left(a_{n}\right)_{0 \leq n \leq N+1}$ is a sequence of elements of the set $\{0,1\}$. By the hypothesis and the induction hypothesis we have $\left|\sum_{n=0}^{N+1} a_{n} y_{n}\right| \leq\left|\sum_{n=0}^{N} a_{n} y_{n}\right|+\left|a_{n+1} y_{n+1}\right|<\frac{1}{3}+\frac{1}{3}$. Since $\lambda s_{N+1}=x+y$, where $x \in \mathbb{Z}$ and $|y|=\left\|\lambda s_{N+1}\right\|<1 / 3$, and $\lambda s_{N+1}=\sum_{n=0}^{N+1} a_{n} x_{n}+\sum_{n=0}^{N+1} a_{n} y_{n}$, we see that $\sum_{n=0}^{N+1} a_{n} y_{n}-y \in \mathbb{Z}$. It follows by the inequalities $\left|\sum_{n=0}^{N+1} a_{n} y_{n}-y\right| \leq\left|\sum_{n=0}^{N+1} a_{n} y_{n}\right|+$ $|y|<\frac{2}{3}+\frac{1}{3}$ that $\sum_{n=0}^{N+1} a_{n} y_{n}-y=0,\left|\sum_{n=0}^{N+1} a_{n} y_{n}\right|=|y|<\frac{1}{3}$, and so (7) is true. Now fix (for a moment) a positive rational integer $N$, and consider the subsets, say $U$ and 
$V$, of $\{0,1, \ldots, N\}$ defined as follows: $n \in U \Leftrightarrow y_{n}>0$, and $n \in V \Leftrightarrow y_{n}<0$. Then, $\sum_{n=0}^{N}\left\|\lambda \theta^{n}\right\|=\sum_{n=0}^{N}\left|y_{n}\right|=\sum_{n \in U} y_{n}+\sum_{n \in V}\left(-y_{n}\right)$, and so

$$
\sum_{n=0}^{N}\left\|\lambda \theta^{n}\right\|<\frac{2}{3},
$$

since by (7) we have $\left|\sum_{n \in U} y_{n}\right|<\frac{1}{3}$ and $\left|\sum_{n \in V}\left(-y_{n}\right)\right|<\frac{1}{3}$. Letting $N$ tend to infinity in (8), we obtain the result by Lemma 1.

\section{REFERENCES}

1. M. J. Bertin, A. Decomps-Guilloux, M. Grandet-Hugo, M. Pathiaux-Delefosse and J. P. Schreiber, Pisot and Salem numbers (Birkhäuser Verlag Basel, 1992).

2. D. W. Boyd, Linear recurrence relations for some generalized Pisot sequences, in Advances in Number Theory, Proceedings of the 1991 CNTA Conference (Gouvea F. Q. and Yui N., Editors) (Oxford University Press, Oxford, UK, 1993), 333-340.

3. A. Dubickas, Even and odd integral parts of powers of a real number, Glasg. Math. J. 48 (2006), 331-336.

4. C. Pisot, La répartition modulo un et les nombres algébriques, Annali Scuola Norm. Sup. Pisa. 7 (1938), 205-248.

5. R. Salem, Algebraic numbers and Fourier analysis (Heath Math. Monographs, Health, Boston, MA, 1963).

6. C. J. Smyth, The conjugates of algebraic integers, Amer. Math. Mont. 82 (1975), 86.

7. T. Zaïmi, Comments on the distribution modulo one of powers of Pisot and Salem numbers, Publ. Math. Debrecen (to appear). 